\title{
An Improved Iterative Technique for the Quasi-TEM Analysis of Generalized Planar Lines
}

\author{
Enrique Drake, Francisco Medina, Member IEEE, and Manuel Horno, Member IEEE
}

\begin{abstract}
The Generalized Bioconjugate Gradient Method (GBGM) and FFT algorithms are used for the quasi-TEM analysis of generalized multistrip lines embedded in multilayered lossless/lossy, iso/anisotropic dielectric and/or magnetic media. Important computational improvement is achieved by including asymptotic extraction techniques in the determination of the spatial Green's function matrix. Comparisons with other iterative procedures are presented. Several practical structures are analyzed and numerical results are compared with previously published data.
\end{abstract}

\section{INTRODUCTION}

$I^{2}$ N THE PAST decades, the quasi-TEM approximation has been extensively used to analyze planar microstriplike lines appearing in MIC and MMIC. As it is well known, quasi-TEM analysis is useful and reasonably accurate at the lower end of the frequency spectrum for many practical lines involving lossless/lossy dielectric/magnetic materials [1].

Under quasi-TEM assumption, the propagation problem can be reduced to solving the two dimensional Laplace's equation subjected to the appropriate boundary conditions. A wide variety of techniques has been used to solve that problem (conformal mapping, spectral and variational methods, integral equation method and so on). When one of these standard methods is applied to the analysis of planar structures of arbitrary geometry, the addition of substrate layers and metallizations considerably complicates the application of the method. This also occurs in the resolution of other electromagnetic problems (scattering, radiation ....) in which planar structures are involved. Owing to this, several iterative procedures have been recently proposed to deal with this type of problems [2]-[8]. These iterative techniques, in conjunction with FFT algorithms, provide an efficient way to solve integral or matrix convolutional equations. In the case of large size matrix operators, the primary advantage arising from the use of recursive algorithms is to circumvent the excessive storage problems inherent in the Gaussian elimination or other direct inversion methods. Another argument for iteratively solving an operator equation is the obvious fact that the process can be stopped once a pre-

Manuscript received May 20, 1991 revised October 28, 1991. This work was supported by the DGICYT, Spain (Project PB87-0798-C03-01).

The authors are with the Microwave Group, Department of Electronics and Electromagnetism, University of Seville, Avda. Reina Mercedes s./n. 41012 Seville, Spain.

IEEE Log Number 9106048. specified degree of accuracy in the solution is reached. This generally results in CPU time savings. In addition, the choice of the initial estimate (starting point of the iterative process) is not critical. Therefore, it is not necessary to have previous knowledge of the features of the solution.

The different versions of the Conjugate Gradient Method (CGM) are probably the best known iterative techniques [6]. In contrast to the spectral iterative techniques [3], [7], the CGM offers theoretical convergence to the exact solution in a finite number of steps (in absence of round-off error). Nevertheless, in some practical cases, the spectral iterative techniques (CCST [3], SIM [7]) have proved to have a higher rate of convergence than the CGM.

A modification of the CGM has been recently developed to enhance its rate of convergence: the Generalized Biconjugate Gradient Method (GBGM) [8]. The GBGM simultaneously solves both the operator equation and its adjoint equation, thus avoiding the resolution of the normal equation associated with non-Hermitian operatorsthis is the case in this paper-, which is one of the main reasons for the slow convergence in the CGM. In the present paper, we intend to use the GBGM for analyzing a very general class of planar transmission lines under quasi-TEM assumption and to compare the GBGM with other iterative schemes.

Prior to solving the integral equation for the unknown free charge density per unit length (p.u.1.) on the conducting strips, it is necessary to determine the spatial Green's function matrix corresponding to the structure under analysis. In this paper, we have also focused our attention on the efficient computation of this quantity. To achieve this goal, we have used an efficient asymptotic extraction technique in the determination of the spatial Green's function from its spectral representation. The spectral Green's function is readily obtained by using the theory explained in [9], [1]. This technique, together with the FFT algorithm, has made it possible to minimize memory storage and CPU time.

In order to illustrate the validity and the strength of the method, numerical results are presented and compared with published data for some practical structures.

\section{Outline of the Problem: Quasi-TEM Analysis}

The cross section of the general planar multiconductor transmission line to be analyzed is shown in Fig. 1. The 




Fig. 1. Cross-section of a general multilayered multistrip line.

system presents translational symmetry in the direction perpendicular to the $x-y$ plane. The stratified medium is made of $N$ layers of lossy iso/anisotropic dielectric or magnetic substrates. The lower boundary of the configuration (interface 0 ) is an electric wall and the upper boundary (interface $N$ ) can be considered to be any one of these three possibilities: grounded plates, magnetic walls or open boundaries. The transverse permittivity tensor $\left[\epsilon_{i}\right]$, and the transverse magnetic permeability tensor $\left[\mu_{i}\right]$ of each layer $(i=1, \cdots, N)$ are assumed to be complex in order to account for substrate losses in the analysis. The equivalent permittivity tensor [1] used for the determination of the inductance matrix, $[L]$, becomes non-symmetrical if longitudinally magnetized semiconductors or ferrites are involved. In Fig. 1, $M$ interfaces $\left(n_{k}, k=1, \cdots, M\right)$ are occupied by an arbitrary number, $N_{c}$, of infinitely thin perfect conducting strips with arbitrary locations.

The determination of the quasi-TEM propagation parameters of the line is entirely based on the evaluation of the complex capacitance matrix per unit length (p.u.l), $[C],[1]$. This evaluation implies the resolution of the following system of integral equations (for $N_{c}$ canonical excitation problems):

$$
\left\{\begin{array}{l}
V_{i}(x)=\sum_{j=1}^{M} \int_{D_{j}} G_{i j}\left(x-x^{\prime}\right) \rho_{j}\left(x^{\prime}\right) d x^{\prime} \quad x \in D_{i} \\
\rho_{i}(x)=0 \quad x \notin D_{i} \quad i=1, \cdots, M
\end{array}\right.
$$

where $D_{i}$ is the region occupied by metallizations at the ith metallized interface, $\rho_{i}(x)$ and $V_{i}(x)$ are the complex charge density and the voltage excitation at the ith metallized interface respectively, and $\mathrm{G}_{\mathrm{ij}}\left(x-x^{\prime}\right)(i, j=1$, ..,$M)$ stand for the values of the spatial Green's function at the metallized interfaces.

\section{ApPlication of the GBGM-FFT Algorithm}

The GBGM [8] is an iterative method used to solve the operator equation $A I=Y$ in which $A$ is a given linear operator and $I$ is the unknown to be found for a particular excitation $Y$. As it is said in [8], the GBGM is specially fitted for the solution of the equation $A I=Y$ when the operator $A$ is non-Hermitian. In general, this is the case of the operator equation appearing in (1) when substrate losses, or longitudinally magnetized semiconductors or ferrites are present. Nevertheless, in the present work we have checked that the GBGM has a faster convergence than the ordinary CGM even if the operator of (1) is Hermitian.

To solve (1) by means of the GBGM, it is necessary to discretize that convolutional expression. Two possibilities are available for this purpose: the use of the Method of Moments (MM) [4], [10] or the direct application of the GBGM. In the present paper, we choose the latter option. The total region that takes part in the problem is divided into $N_{p}$ subintervals of width $T$. All the functions which are defined in that region and appear in the iterative process (including the charge density and the Green's functions) are considered to be constant in each subinterval and are assumed to be equal to their value at the center of the subregion. Once the discretization process has been carried out, in each iteration, the convolutions are evaluated at the same points at which the original functions are sampled. This is what a method of moment practitioner would term as delta function expansion and weighting.

At this point, it must be noted that in order to compute a linear convolution sum in an efficient way, it is suitable to approximate that linear convolution by a cyclic discrete convolution, thus taking advantage of the use of FFT algorithms. After doing this, (1) is reduced to

$$
\begin{array}{r}
V_{i}(k T)=T \mathrm{FFT}^{-1}\left\{\sum_{j=1}^{M} \tilde{G}_{i j}^{\prime}(n) \mathrm{FFT}\left\{\rho_{j}\right\}\right\} \\
\forall k / k T \in D_{i} \quad i=1, \cdots, M
\end{array}
$$

where $V_{i}(k T)$ is the voltage (with value 0 or 1 ) on the $k$ th point sampled on the strips of the $i$ th metallized interface, FFT $\left\{\rho_{j}\right\}$ is the Fast Fourier Transform of the sampled charge density at the jth metallized interface including the zero padding for the regions without metallizations, and the $\tilde{G}_{i j}^{\prime}(n)(i, j=1, \cdots, M)$ are obtained as described in the following section. Once the discretization process has been carried out, the computational implementation of the GBGM is no longer a problem because (2) is just a system of a linear algebraic equations.

It can be observed that the use of FFT (corresponding to cyclic convolutions) to compute linear convolution sums implies that the cross section of the line under study presents a periodic nature (in the $x$-axis direction). In fact, if $T_{0}$ is the total width of the sampled region $\left(T_{0}=N_{p} T\right)$, the equation (2) corresponds to the structure obtained by the periodic repetition of that region with period $T_{0}$. Therefore, the aperiodic sections must be periodically simulated by introducing two fictitious side walls far away from the metallized regions. As we will see, the choice of the width $\left(T_{0}\right)$ of an appropriate simulating period is a function of the geometrical characteristics of each line. Obviously, really periodic structures are taken into account in an exact way. 


\section{Treatment of the Green's Function Matrix}

The computation of the spatial Green's function matrix for a general multilayered configuration cannot be achieved in closed form. On the contrary, a very simple systematic algorithm can be implemented to obtain its Fourier transform. This has been done here by using the recurrent scheme reported in [9]-valid for non-coplanar conducting strips-in conjunction with the theory developed in [1] - which enables us to deal with lossy and magnetic substrates. This technique has been recently called the Equivalent Boundary Method (EBM) [11].

In practice, the efficient computation of the convolution sums is achieved by using the Discrete Convolution Theorem and the FFT algorithms. The application of this technique only requires the knowledge of the spectral Green's function matrix. However, a computational question drives us to build an approximation of the spatial Green's function matrix. When the periodic simulation of an aperiodic structure is performed, all the discretized functions must be usually padded with a large number of zeros. This zero padding may force us to store an excessive amount of samples with the consequent problems of CPU time and memory storage limitation. The knowledge of an approximation of the spatial Green's function matrix would allow us to overcome this drawback by keeping only the part of it which is involved in the convolution process, i.e., a middle region whose width is twice the total width of the region with metallizations.

As a first possibility, we might sample the spectral Green's functions $\left\{\tilde{G}_{i j}(\alpha)\right\}_{i, j=1}^{M}$ and apply the adequate inverse FFT's. However, the band-unlimited character of these spectral functions, specially when $i=j$, would force us to keep a high number of samples to reduce the inherent error associated with the spectral truncation. In the present paper, a new asymptotic extraction technique has been applied to the diagonal spectral Green's function $\left\{\tilde{G}_{i i}(\alpha)\right\}_{i=1}^{M}$ to minimize the storage requirements and the CPU time of these inverse FFT's. Off-diagonal elements have not been treated since they exponentially approach to zero when the spectral variable, $\alpha$, approaches infinity.

From the studies presented in [9] and [1], it is easy to check that the asymptotic behavior of the diagonal spectral Green's function associated with each metallized interface $(i=1, \cdots, M)$ is

$$
\tilde{G}_{i i}(\alpha) \rightarrow \frac{K_{ \pm}^{i}}{|\alpha|} \quad \text { for } \alpha \rightarrow \pm \infty
$$

where

$$
K_{ \pm}^{i}=\frac{1}{ \pm j K^{i}+\epsilon_{y y}^{i} S_{i}+\epsilon_{y y}^{i+1} S_{i+1}}
$$

$j$ being the imaginary unit $\sqrt{-1}$, and

$$
K^{i}=\frac{\epsilon_{y x}^{i}+\epsilon_{x y}^{i+1}-\epsilon_{x y}^{i}-\epsilon_{y x}^{i+1}}{2}
$$

$$
\begin{aligned}
& S_{i}=\left(\frac{\epsilon_{x x}^{i}}{\epsilon_{y y}^{i}}-R_{i}^{2}\right)^{1 / 2} \\
& R_{i}=\frac{\epsilon_{x y}^{i}+\epsilon_{y x}^{i}}{2 \epsilon_{y y}^{i}} \quad(i=1, \cdots, M) .
\end{aligned}
$$

Observe that when any substrate adjacent to the metallizations has a complex non-symmetrical permeability tensor and, therefore, a complex non-symmetrical equivalent permittivity tensor, the asymptotic behavior of the corresponding Green's function has not any symmetry with respect to the spectral variable $\alpha$ (in this sense, we have in general a non-symmetrical spectral Green's function).

In the following, we are going to define auxiliary functions $\tilde{G}_{a s}^{i}(\alpha)$ associated with the diagonal spectral Green's functions $\tilde{G}_{i i}(\alpha)$. The functions $\tilde{G}_{a s}^{i}(\alpha)$ and $\tilde{G}_{i i}(\alpha)$ must have the same asymptotic behavior in the spectral domain for a given value of $i$. In addition, the spatial counterpart of $\tilde{G}_{a s}^{i}(\alpha)$ must be analytically known. In the application of the asymptotic extraction technique the spectral Green's function matrix is first obtained by using the EBM. Then, the auxiliary $\tilde{G}_{a s}^{i}(\alpha)$ are substracted from the diagonal $\tilde{G}_{i i}(\alpha)$ for $i=1, \cdots, M$.

Let $\tilde{G}_{a s}^{i}(\alpha)$ be the spectral Green's function at the ith metallized interface corresponding to the structure obtained from the original line by removing the upper boundary and replacing the original substrates by an isotropic and homogeneous medium with dielectric permittivity $\epsilon_{a s}^{i}$. The analytical expression of $\tilde{G}_{a s}^{i}(\alpha)$ may be easily obtained from the EBM [9]:

$$
\tilde{G}_{a s}^{i}(\alpha)=\left[\epsilon_{a s}^{i}\left(|\alpha|+\alpha \operatorname{coth}\left(\alpha h_{a s}^{i}\right)\right)\right]^{-1}
$$

where $\epsilon_{a s}^{i}$ must be chosen in such a way that the possible non-symmetrical asymptotic behavior of $\tilde{G}_{i i}(\alpha)$ is accommodated, i.e.:

$$
\epsilon_{a s}^{i}= \begin{cases}\epsilon_{+}^{i}=\frac{1}{2 K_{+}^{i}} & \alpha>0 \\ \epsilon_{-}^{i}=\frac{1}{2 K_{-}^{i}} & \alpha<0 \\ \epsilon_{o}^{i}=\frac{\epsilon_{+}^{i}+\epsilon_{-}^{i}}{2} & \alpha=0\end{cases}
$$

and the effective substrate height $h_{a s}^{i}$, although arbitrary to some extent, has been chosen in such a way that the condition $\operatorname{Re}\left\{\tilde{G}_{a s}^{i}(0)\right\}=\operatorname{Re}\left\{\tilde{G}_{i i}(0)\right\}$ is fulfilled. With this choice, $\tilde{G}_{a s}^{i}(\alpha)$ and $\tilde{G}_{i i}(\alpha)$ are not very different in the surroundings of $\alpha=0$, thus avoiding numerical problems as we will see later on.

At this point, we can obtain a function matrix $\left[\tilde{G}_{d}^{i j}(\alpha)\right](i, j=1, \cdots, M)$ defined as follows:

$$
\tilde{G}_{d}^{i j}(\alpha)=\left\{\begin{array}{ll}
\tilde{G}_{i j}(\alpha)-\tilde{G}_{a s}^{i}(\alpha) & \text { for } i=j \\
\tilde{G}_{i j}(\alpha) & \text { for } i \neq j
\end{array} .\right.
$$


A discrete approximation of the corresponding spatial function matrix $\left[G_{d}\left(x-x^{\prime}\right)\right]$ can be built by taking $N_{p}$ samples (with period equal to $1 / T_{0}$ ) of $\left[\tilde{G}_{d}(\alpha)\right]$ and applying inverse FFT:

$$
\begin{gathered}
G_{d}^{i j}(m T)=\frac{1}{T} \mathrm{FFT}^{-1}\left\{\tilde{G}_{d}^{i j}\left(n / T_{0}\right)\right\} \\
m, n=-N_{p} / 2, \cdots, N_{p} / 2-1 \\
i, j=1, \cdots, M .
\end{gathered}
$$

As a consequence of the asymptotic extraction process, the functions matrix $\left[\tilde{G}_{d}(\alpha)\right]$ has a narrower range of values significantly different from zero, thus making possible the drastic reduction of the number $N_{p}$ of samples. This reduction of $N_{p}$ and the consequent diminution of the size of the sampled spectral region $\left(N_{p} / T_{0}=1 / \mathrm{T}\right)$ imply a larger separation $(T)$ between the contiguous samples in the spatial domain. Third order spline interpolation is now used to increase the discretization level. Once the samples of $\left[G_{d}\right]$ in (8) have been interpolated with an interpolation factor of $N_{i}$, we have $N_{p i}=N_{i} N_{p}$ points of $\left[G_{d}\left(x-x^{\prime}\right)\right]$ separated by a period $T_{i}=T / N_{i}$. At this point, it is important to remember that only the $N_{c}$ samples corresponding to a middle interval-whose width is twice the total width of the region with metallizations-are going to be involved in the convolutions. Hence, only $N_{c}$ samples of the discretized spatial Green's functions $\mathrm{G}_{\mathrm{ij}}\left(\mathrm{mT}_{\mathrm{i}}\right) m=$ $-N_{c} / 2, \cdots, N_{c} / 2-1$ must be computed from $G_{d}^{i j}\left(m T_{i}\right)$ and $G_{a s}^{i}\left(m T_{i}\right)$ :

$$
G_{i j}\left(m T_{i}\right)= \begin{cases}G_{d}^{i j}\left(m T_{i}\right)+G_{a s}^{i}\left(m T_{i}\right) & \text { for } i=j \\ G_{d}^{i j}\left(m T_{i}\right) & \text { for } i \neq j\end{cases}
$$

where $G_{a s}^{i}\left(m T_{i}\right)$ are samples of $G_{a s}^{i}\left(x-\mathrm{x}^{\prime}\right)$ which are the inverse Fourier transforms of infinite combs of samples of $G_{a s}^{i}(\alpha)$ taken with period equal to $1 / T_{0}$. Note that the functions $G_{a s}^{i}\left(x-x^{\prime}\right)$ computed in this way are the spatial Green's functions of the asymptotic equivalent structures keeping the spatial periodicity (with period $T_{0}$ ) in the $x$-direction. The functions $G_{a s}^{i}\left(x-x^{\prime}\right)(i=1, \cdots, M)$ have been analytically obtained as

$$
\begin{aligned}
G_{a s}^{i}\left(x-x^{\prime}\right) & \\
= & G_{o}^{i}+\frac{1}{4 \pi \epsilon_{+}^{i}} \ln \left\{A^{i}+j B^{i} \cot \left(\frac{\pi\left(x-x^{\prime}\right)}{T_{0}}\right)\right\} \\
& +\frac{1}{4 \pi \epsilon_{-}^{i}} \ln \left\{A^{i}-j B^{i} \cot \left(\frac{\pi\left(x-x^{\prime}\right)}{T_{0}}\right)\right\}
\end{aligned}
$$

where

$$
\begin{aligned}
G_{o}^{i} & =\frac{h_{a s}^{i}}{2 T_{0}}\left(\frac{1}{\epsilon_{+}^{i}}+\frac{1}{\epsilon_{-}^{i}}\right) \\
A^{i} & =\frac{1+\exp \left(-4 \pi h_{a s}^{i} / T_{0}\right)}{2} \\
B^{i} & =\frac{1-\exp \left(-4 \pi h_{a s}^{i} / T_{0}\right)}{2} \quad i=1, \cdots, M .
\end{aligned}
$$

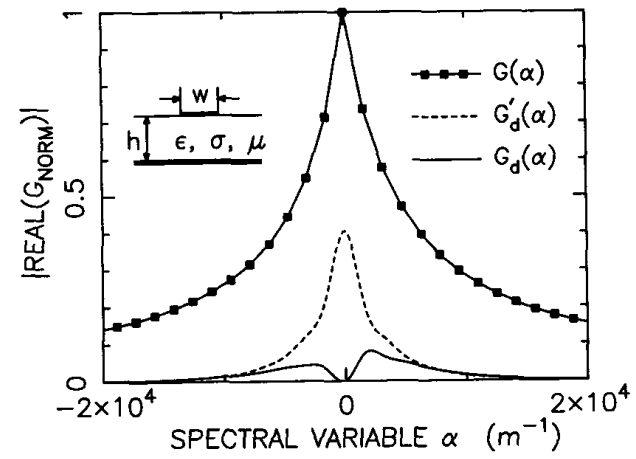

(a)

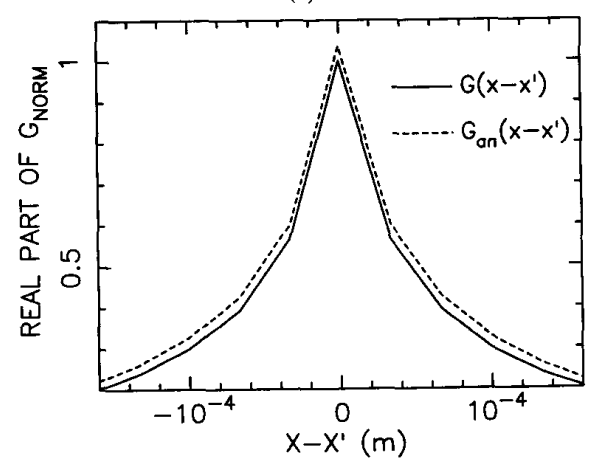

(b)

Fig. 2. (a) The spectral Green's function $G$ and the remainder spectral functions after asymptotic extraction $\tilde{G}_{d}^{\prime}$ (with $h_{a s}=\mathrm{h}$ ) and $\tilde{G}_{d}$ (with $h_{a s}$ as in this work). Note the nonsymmetrical nature of the Green's function with respect to $\alpha$. (b) The middle region of the spatial Green's function $G$ and its analytical part $G_{a n}$ for a microstrip configuration on saturated FMS ( $h$ $=100 \mu \mathrm{m}, w=200 \mu \mathrm{m}, \epsilon=15 \epsilon_{o}, \sigma=5(\Omega \mathrm{m})^{-1}, 4 \pi M_{s}=2000 \mathrm{G}, H_{o}=$ $1500 O e, \Delta H=75 O e)$

The singularities of $G_{i j}(0)$ have been replaced by the numerically computed integral averages of $G_{i j}\left(x-x^{\prime}\right)$ in the central interval $[-T / 2, T / 2]$. Finally, the values of $\tilde{G}_{i j}^{\prime}(n)$ of (2) are worked out from the $N_{c}$ samples of the spatial Green's functions $G_{i j}^{\prime}(m)$ (where the prime mark denotes the replacement of $\left.G_{i j}(0)\right)$ by direct FFT's:

$$
\begin{gathered}
\tilde{G}_{i j}^{\prime}(n)=\operatorname{FFT}\left\{G_{i j}^{\prime}\left(m T_{i}\right)\right\} \\
m, n=-N_{c} / 2, \cdots, N_{c} / 2-1 \\
i, j=1, \cdots, M
\end{gathered}
$$

Once the functions $\tilde{G}_{i j}^{\prime}(n)$ have been computed, (2) is ready to be solved by GBGM. Since an interpolation process is assumed, we must substitute $T$ by $T_{i}$ in (2).

\section{Numerical Results}

To illustrate the asymptotic extraction technique described above, the Fig. 2(a) shows the absolute value of the real part of the normalized spectral Green's function $\tilde{G}(\alpha)$ of a microstrip configuration on saturated FMS substrate longitudinally magnetized. Notice that the presence of an external longitudinal magnetic field $H_{o}$ makes the spectral Green's function be non-symmetrical with re- 


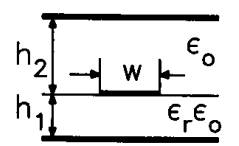

(1)

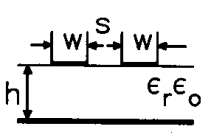

(II)



(III)

(a)

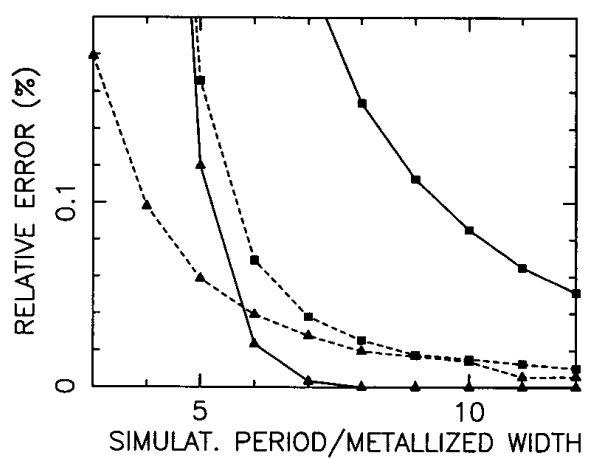

(b)

Fig. 3. (a) Several microstrip configurations on a dielectric substrate of $\epsilon_{r}$ $=15$. (b) Relative error in the self-capacitance of a conducting strip with the configurations of Fig. 3(a) versus the ratio between the simulating period and the width of the metallized region. (1) with $w / h_{1}=1, h_{2} / h_{1}=20$; ( $1, h_{2} / h_{1}=1 ;(-\Delta-\Delta-\Delta-\Delta-\Delta-)$ for (II) with $w / h=1, s / h=0.1$; (-口-) for (III) with $w / h_{1},=1, h_{2} / h_{1}=0.1$.

spect to $\alpha$. If we apply the asymptotic extraction scheme with the choice $h_{a s}=h$, the dashed line is obtained for the remainder Green's function $\tilde{G}_{d}^{\prime}(\alpha)$. It must be remarked that the sharp nature of $\tilde{G}_{d}^{\prime}(\alpha)$ would force us to have fine sampling. Because of this, the choice of $h_{a s}$ that makes $\operatorname{Re}\left(\tilde{G}_{a s}(0)\right)=\operatorname{Re}(\tilde{G}(0))$ is more suitable (solid line). Another advantage of the asymptotic extraction technique is that the central region of the spatial Green's function $G\left(x-x^{\prime}\right)$, involved in the convolution process, is almost analytically built up (see Fig. 2(b)). The values of the Green's function in that region are mainly affected by the spectral asymptotic behavior, which is analytically taken into account.

In a previous section, we have justified the need to select a simulating period for the analysis of aperiodic structures. In the present work, we have investigated the relation between the suitable size of the periodic window and the features of the line studied. Fig. 3(b) shows the relative error introduced for the periodic simulation in the self-capacitance of a conducting strip under different configurations (see Fig. 3(a)) as a function of the ratio between the width of the simulating periodic window and the width of the metallized region. The error is relative to the value of the self-capacitance when the simulating pe-

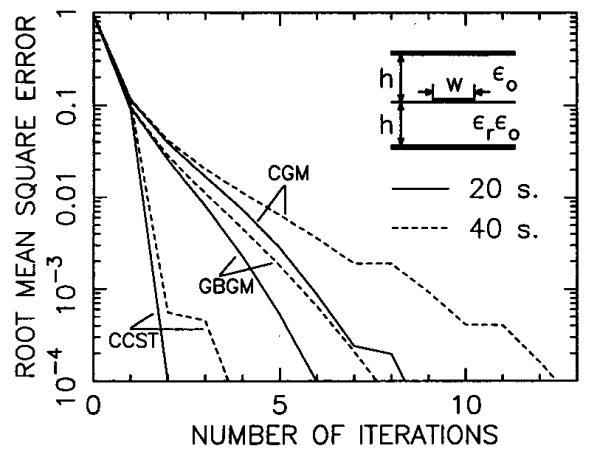

(a)

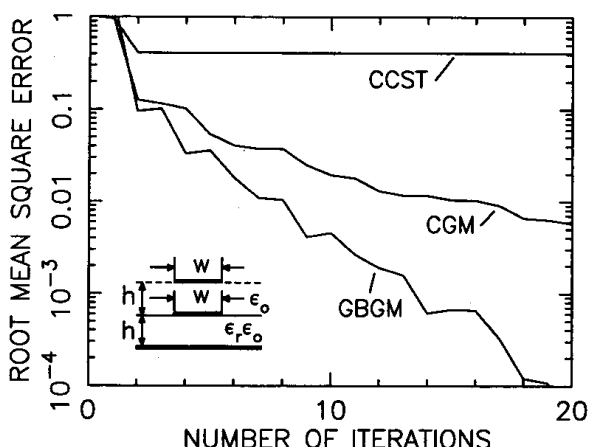

(b)

Fig. 4. Rates of convergence of different iterative algorithms for the calculation of (a) the capacitance of a symmetrical stripline on alumina $\left(\epsilon_{r}=\right.$ $9.6, w / h=1)$ with 20 or 40 samples on the strip and (b) the self-capacitance of the lower strip of a broadside configuration $\left(\epsilon_{r}=15, w / h=1\right)$.

riod approaches inifinity. Notice that the relative proximity between conductors (specially, grounded plates) implies a closer confinement of the electromagnetic field to the metallized region, thus allowing us to reduce the width of the periodic window.

Another important aspect is to check the improvement introduced in the rate of convergence of the iterative process by the use of the GBGM instead of the ordinary CGM algorithm. A spectral iterative technique successfully used in [3] (named CCST) has been also programmed for comparison. In Fig. 4(a), we compare the rates of convergence of the CGM, the GBGM and the CCST in the computation of the capacitance per unit length of a symmetrical stripline. These results correspond to both 20 and 40 samples on the strip. The $50 \%$ reduction in the number of iterations obtained by using the GBGM instead of CGM is a very typical result in the structures analyzed. Anyway, the highest rate of convergence corresponds to the CCST. Nevertheless, Fig. 4(b) repeats the comparison for a pair of broadside coupled strips, showing the stagnation process (one-step improvement is less than the computer precision) in the CCST. The stagnation problem in the spectral iterative techniques was observed in [7]. This led the authors of that paper to modify the algorithm. In relation to the CPU time, the GBGM presented an average 


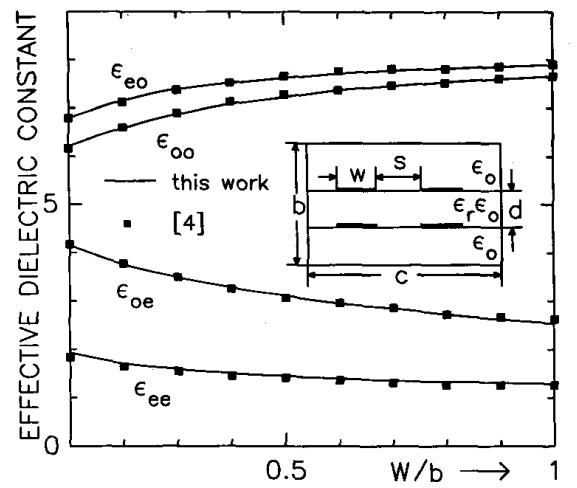

Fig. 5. Effective dielectric constants of a broadside, edge-coupled microstrip with inverted dielectric in $[4](s / b=d / b=0.2, c / b=10$, $\epsilon_{r}=10$ ).

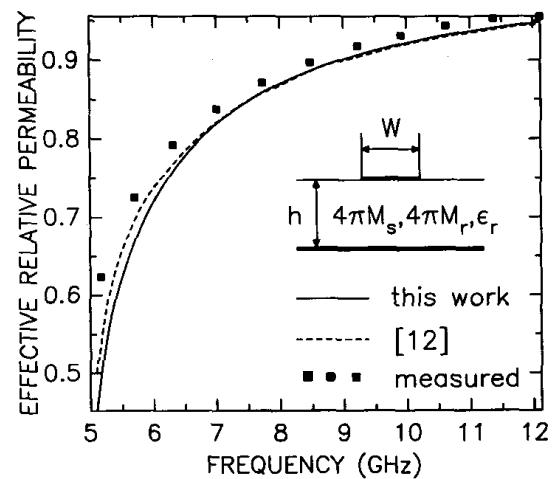

(a)

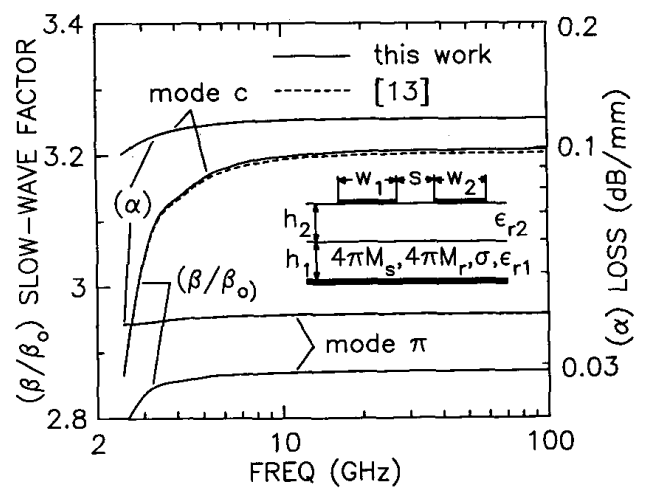

(b)

Fig 6. (a) Effective relative permeability of a microstrip on a latched garnet substrate in $[12]\left(W / h=0.5,4 \pi M_{s}=1780 \mathrm{G}, 4 \pi M_{r}=1030 \mathrm{G}\right)$. (b) Modal slow-wave factors and attenuation constants for a pair of asymmetrical strips on two layers in the partially demagnetized state (see [13]) $\left(h_{1}=100 \mu \mathrm{m}, h_{2}=100 \mu \mathrm{m}, w_{1}=160 \mu \mathrm{m}, s=100 \mu \mathrm{m}, w_{2}=100 \mu \mathrm{m}\right.$, $\epsilon_{r 1}=\epsilon_{r 2}=14.9,4 \pi M_{s}=870 \mathrm{G}, 4 \pi M_{r}=550 \mathrm{G}$, dielectric losses: $\sigma=$ $0.001(\Omega \mathrm{mm} .)^{-1}$, magnetic losses: $\left.A=0.01, N=1.5\right)$.

of $20 \mathrm{~ms}$ per iteration (in the case of 40 samples) on a VAX-11/785 computer, while the other two algorithms presented $60 \mathrm{~ms}$ per iteration.

Finally, in Figs. 5 and 6 , we include the analysis of some practical structures for comparison purpose. The results are compared with data reported in the bibliography, and very good agreement is found. The structure analyzed in Fig. 5 is an example of multiconductor configuration with non-coplanar metallizations. Symmetry of this structure is not taken into account because our aim is to check the efficiency of the algorithm programmed for the case of several metallized interfaces. In Fig. 6, a pair of configurations with gyromagnetic substrates are considered (non-symmetrical spectral Green's functions are involved).

The method has been exhaustively checked by comparing it with many other data reported in the literature with similar result. The convergence of the method in complex cases involving multilayered, lossy and anisotropic materials has been also verified. We conclude that the results obtained with the computer programs based on the theory in this paper are accurate and reliable as long as quasiTEM approximation remains valid. So, this method is an efficient alternative to other methods (for example the method of moments) applied to the quasi-TEM analysis of very general planar structures.

\section{Conclusion}

In this paper, we have presented the quasi-TEM analysis of a wide class of planar multiconductor transmission lines by employing the GBGM and FFT algorithms. Printed conductors are embedded in a layered structure including dielectrics, semiconductors or magnetic materials. Natural anisotropy and anisotropy produced by longitudinal magnetizing fields are accounted for in the analysis.

The spatial Green's function matrix is used in the formulation of the problem to reduce memory storage and CPU time. This matrix is obtained for the multilayered structure from its spectral domain representation, which can be readily computed by means of a simple recurrent scheme (EBM). This process has been significantly accelerated by using an asymptotic extraction technique in the spectral domain. The singular behavior of the spatial domain Green's matrix is analytically taken into account in such a way that the remainder spectral matrix is a narrow band function. In particular, the presence of longitudinally magnetized ferrites or semiconductors-which results in non-symmetrical spectral Green's functionscan be accommodated by using this method.

Several aspects related to the convergence bahavior of the method have been investigated. The choice of simulating periods to analyze aperiodic lines has been found to be strongly related to the geometrical features of the lines. The superiority (in the sense of a faster rate of convergence) of the GBGM over the ordinary CGM algorithm has also been checked. In spite of the fact that CCST has proved to have the highest rate of convergence, it presents some stagnation problems. Some examples have been included to illustrate the strength and the versatility of the method. Comparisons with published data indicate that the method presented yields accurate results, thus offering an efficient alternative technique for the quasi-TEM analysis of planar lines. 


\section{REFERENCES}

[1] M. Horno, F. L. Mesa, F. Medina, and R. Marqués, "Quasi-TEM analysis of multilayered, multiconductor, coplanar structures with dielectric and magnetic anisotropy including substrate losses," IEEE Trans. Microwave Theory Tech., vol. 38, pp. 1059-1068, Aug. 1990.

[2] T. K. Sarkar and S. M. Rao, "An iterative method for solving electrostatic problems," IEEE Trans. Antennas Propagat., vol. AP-30, no. 4, pp. 611-616, 1982

[3] P. M. van den Berg, "Iterative computational techniques in scattering based upon the integrated square error criterion," IEEE Trans. Antennas Propagat., vol. AP-32, pp. 1063-1071, Oct. 1984.

[4] C. H. Chan and R. Mittra, "Analysis of MMIC structures using an efficient iterative approach," IEEE Trans. Microwave Theory Tech., vol. 36, pp. 96-105, Jan. 1988.

[5] P. M. van den Berg, "Iterative schemes based on the minimization of the error in field problems," Electromagn., vol. 5, pp. 237-262, 1985.

[6] T. K. Sarkar and E. Arvas, "On a class of finite step iterative methods (conjugate directions) for the solution of án operator equation arising in Electromagnetics," IEEE Trans. Antennas Propagat., vol. AP-33, pp. 1058-1066, Oct. 1985.

[7] R. Mittra and C. H. Chan, "Iterative approaches to the solution of electromagnetic boundary value problems," Electromagn., vol. 5, no. $2-3$, pp. 123-146, 1985.

[8] T. K. Sarkar, "On the application of the Generalized Biconjugate Gradient Method," J. Electromagn. Wav. Appl., vol. 1, no. 3, pp. 223-242, 1987.

[9] F. Medina and M. Horno, "Determination of Green's function matrix for multiconductor and anisotropic multidielectric planar transmission lines: a variational approach," IEEE Trans. Microwave Theory Tech., vol. MTT-33, pp. 933-940, Oct. 1985.

[10] C. H. Chan and R. Mittra, "Analysis of a class of cylindrical multiconductor transmission lines using an iterative approach," IEEE Trans. Microwave Theory Tech., vol. MTT-35, pp. 415-424, Apr. 1987.

[11] F. Mesa, R. Marqués, and M. Horno, " A general algorithm for computing the bidimensional spectral Green's dyade in multilayered complex bianisotropic media: the Equivalent Boundary Method (EBM)," IEEE Trans. Microwave Theory Tech., vol. MTT-39, pp. 1640-1649, Sept. 1991.

[12] D. J. Masse and R. A. Pucel, "Microstrip propagation on magnetic substrates. Part II: Experiment," IEEE Trans. Microwave Theory Tech., vol. MTT-20, pp. 309-313, May 1972.

[13] F. Mesa and M. Horno, "Quasi-TEM and full wave approaches for coplanar multistrip lines including gyromagnetic media longitudinally magnetized." Microwave Opt. Tech. Lett., vol. 4, pp. 531-534, Nov. 1991.

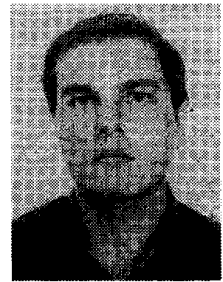

Enrique Drake was born in Montilla, Córdoba, Spain, in September 1966. He received the degree of Licenciado in physics in September 1990 from the University of Seville, Spain.

$\mathrm{He}$ is currently following a $\mathrm{Ph}$.D. program in microwaves with a scholarship of the Spanish Government. His research interests focus on iterative methods for planar structures and multiconductor lines.



Francisco Medina (M'90) was born in Puerto Real, Cádiz, Spain, in November 1960 . He received the Licenciado degree in September 1983 and the Doctor degree in 1987, both in Physics, from the University of Seville, Spain.

$\mathrm{He}$ is currently Associate Professor of Electricity and Magnetism in the Department of Electronics and Electromagnetics, University of Seville. His research deals mainly with analytical and numerical methods for planar structures and multiconductor lines.

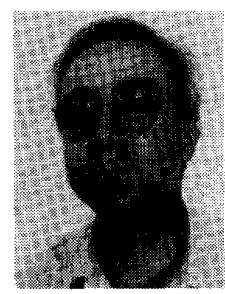

Manuel Horno (M'75) was born in Torre del Campo, Jaén, Spain. He received the degree of Licenciado in physics in June 1969 , and the degree of Doctor in physics in January 1972, both from the University of Seville, Spain.

Since October 1969 he has been with the Department of Electronics and Electromagnetism at the University of Seville, where he became an Assistant Professor in 1970, Associate Professor in 1975, and Professor in 1986. He is a member of Electromagnetism Academy of MIT (Cambridge). His main fields of interest include boundary value problems in electromagnetic theory, wave propagation through anisotropic media, and microwave integrated circuits. He is presently engaged in the analysis of planar transmission lines embedded in anisotropic materials, multiconductor transmission lines, and planar slow-wave structures. 